\title{
Catálogo de las colecciones de líquenes de la región de Magallanes y Antártica Chilena depositadas en el herbario del Instituto de la Patagonia (HIP)
}

\author{
Catalog of the collections of lichens of the Magallanes region (Chile) \\ deposited in the herbarium of the Instituto de la Patagonia (HIP)
}

Javiera Pineda Cáceres ${ }^{1}$, Susana Morano Büchner ${ }^{2} \&$ Osvaldo J. Vidal ${ }^{2 \bowtie}$

\section{Resumen}

La micobiota liquénica de la región de Magallanes es diversa, pero ha sido muy poco estudiada. Esta situación puede redundar en una baja valoración de esta diversidad, junto con un manejo inadecuado para la conservación de este grupo. Se elaboró un catálogo preliminar de la micobiota liquénica de la Región de Magallanes y Antártica Chilena a partir de registros depositados en el Herbario del Instituto de la Patagonia (HIP). El catálogo incluye información sobre hábitat, localidades de colecta, distribución en Chile, distribución global y números de los 247 exsiccata examinados. Se describe información taxonómica, geográfica y ecológica para 100 taxa distribuidos en 3 clases, 9 órdenes, 19 familias y 43 géneros. Los órdenes taxonómicos más diversos fueron Lecanorales (Lecanoromycetes) con 24 géneros y 51 taxa, y Peltigerales (Lecanoromycetes) con 9 géneros y 37 taxa. Estas especies representan colectas realizadas a través de un amplio rango geográfico y ecológico que incluye los 4 distritos provinciales (Magallanes, Tierra del Fuego, Última Esperanza y Antártica Chilena) y ecosistemas estepáricos, arbustivos, boscosos y altoandinos. Esta información puede contribuir a mejorar y sistematizar el actual conocimiento de la micobiota liquénica de la región más austral de Chile para fines de manejo y conservación.

Palabras clave:

herbario, checklist, index herbariorum, Chile, diversidad de líquenes.

\section{Abstract}

The lichen mycobiota of Magellan region is diverse but has been poorly studied. This situation can result in a low rating for this biodiversity and an inadequate management for the conservation of this group. A preliminary catalog of the lichen mycobiota of the Magallanes and Chilean Antarctic region was prepared from records deposited in the Herbarium of the Instituto de la Patagonia (HIP). The catalog includes information on habitat, collection locations, distribution in Chile, global distribution and record numbers of the 296 exsiccata examined. Taxonomical, geographical and ecological information is described for 100 taxa distributed in 3 classes, 9 orders, 19 families and 43 genera. The most diverse taxonomic orders were Lecanorales (Lecanoromycetes) with 24 genera and 51 taxa, and Peltigerales (Lecanoromycetes) with 9 genera and 37 taxa. These species represent collections made through a wide geographical and ecological range including four provincial districts (Magallanes, Tierra del Fuego, Última Esperanza and Chilean Antarctic), and ecosystems like steppes, shrubs, forests and Andean habitats. This information can contribute to improve and systematize the current knowledge of the lichen mycobiota of the southernmost region in Chile for management and conservation purposes.

Facultad de Ciencias Forestales y Recursos Naturales, Universidad Austral de Chile

2 Laboratorio de Botánica, Instituto de la Patagonia,

Universidad de Magallanes

\osvaldo.vidal@umag.cl 


\section{Key words:}

herbarium, checklist, index herbariorum, Chile, lichen diversity.

\section{INTRODUCCIÓN}

Las investigaciones sobre líquenes en Chile son escasas, pese a su alto potencial como uno de los países con mayor diversidad de micobiota liquénica en el mundo. A nivel país se dispone de la "Lista patrón de los líquenes y hongos liquenícolas de Chile" que incluye tanto territorio continental, como antártico e insular (Galloway \& Quilhot, 1998). Por otra parte, existen estudios sobre la flora liquénica de la región de Aysén (Quilhot et al. 2012) y sobre ecosistemas y zonas específicas de Chile, como turberas (Villagra et al. 2009) y parques nacionales (Rubio et al. 2013).

La Región de Magallanes y Antártica Chilena se localiza en el extremo sur del país y del continente sudamericano. Se caracteriza por poseer una amplia y contrastada variabilidad de flora y paisaje vegetal, producto de las condiciones físicas que genera la extensión de la cordillera de los Andes rumbo al sudeste, culminando en el archipiélago del cabo de Hornos (Pisano, 1977). Hasta la fecha no se ha publicado un catálogo completo de la flora liquénica de la región de Magallanes, lo que evidencia un limitado conocimiento sobre estos organismos.

Este estudio tiene por objetivo elaborar un catastro de la flora liquénica depositada en el Herbario del Instituto de la Patagonia, Región de Magallanes y Antártica Chilena. Así, se pretende contribuir en el conocimiento sobre este territorio y la micobiota que alberga, y proveer información básica sobre la distribución de estos organismos, además realizar un correcto y actualizado uso de su nomenclatura y taxonomía.

\section{MATERIALES Y MÉTODOS}

Para confeccionar el catálogo preliminar de flora liquénica de la Región de Magallanes y Antártica Chilena se recopiló información sobre taxa registrados dentro de las cuatro provincias de dicha zona (Magallanes, Última Esperanza, Tierra del Fuego y Antártica Chilena). Los 247 ejemplares estudiados provienen de la colección de líquenes del Herbario del Instituto de la Patagonia (HIP) registrado en el Index Herbarorium del New York Botanical Garden. Estos especímenes fueron identificados por diferentes especialistas al momento de la colecta o posterior a ésta.

Cada taxón reúne datos, obtenidos de las etiquetas de registro, sobre el hábitat (comentarios del registro biológico) y ocurrencia dentro de la región, además de todos los antecedentes disponibles sobre su distribución en Chile y el mundo. Cabe destacar que la información asociada al hábitat en el catálogo no corresponde al hábitat general del taxón, sino a la descripción del hábitat observada al momento de la colecta. La información contenida en el documento Líquenes de Aisén, sur de Chile (Quilhot et al. 2012) fue utilizada como base para este catálogo $\mathrm{y}$, posteriormente, complementada con la literatura correspondiente.

La nomenclatura, taxonomía y autoría de nombres científicos se verificó mediante las bases de datos Index Fungorum (Crous et al. 2004a) y MycoBank (Crous et al. 2004b). Algunos taxa registrados contienen además su nombre sinónimo, debido a que así son referidos en la literatura. Por lo general, cada muestra colectada corresponde a un taxón en particular, pero en ocasiones éstas pueden incluir dos o tres taxa diferentes. En estos casos, se menciona la muestra seguido de un asterisco para su distinción.

Los especímenes se organizaron según su secuencia taxonómica, i.e. la Clase a la cual pertenecen (Arthoniomycetes, Eurotiomycetes y Lecanoromycetes) y con respecto a las demás categorías taxonómicas (orden, familia, género, especie), éstas se ordenaron alfabéticamente dentro de dicha división.

\section{RESULTADOS}

Del total de ejemplares disponibles se identificaron 100 taxa diferentes, organizados en 3 clases, 9 órdenes, 19 familias y 43 géneros (Tabla 1). Lecanoromycetes es la clase más diversa con 17 familias en 7 órdenes, conteniendo 41 géneros y 98 taxa. Su orden más rico es Lecanorales comprendiendo 51 taxa y le sigue Peltigerales con 37 taxa. Los 296 especímenes depositados del HIP se distribuyen al interior de las cuatro provincias de la región. La provincia con más registros fue 
Tabla 1. Sistemática de la flora liquénica total.

\begin{tabular}{|c|c|c|c|c|}
\hline Clases & Órdenes & Familias & Géneros & Taxa \\
\hline Arthoniomycetes & Arthoniales & 1 & 1 & 1 \\
\hline \multirow[t]{4}{*}{ Eurotiomycetes } & Verrucariales & 1 & 1 & 1 \\
\hline & Caliciales & 1 & 2 & 2 \\
\hline & Lecanorales & 7 & 24 & 51 \\
\hline & Ostropales & 1 & 1 & 1 \\
\hline \multirow[t]{5}{*}{ Lecanoromycetes } & Peltigerales & 4 & 9 & 37 \\
\hline & Pertusariales & 2 & 2 & 4 \\
\hline & Teloschistales & 1 & 2 & 2 \\
\hline & Umbilicariales & 1 & 1 & 1 \\
\hline & Total & 19 & 43 & 100 \\
\hline
\end{tabular}

la de Magallanes con 104 ejemplares, le sigue Última Esperanza con 102, Tierra del Fuego con 56 y, por último, Antártica Chilena con 34 muestras colectadas. A continuación, se presenta el catálogo de especies según su respectiva secuencia taxonómica:

Arthoniomycetes: Arthoniales

Chrysotrichaceae

Chrysothrix pavonii (Fr.) J.R. Laundon

Hábitat: bosque, abundante en el bosque.

Registros en Magallanes: río Bueno, Forestal Trillium, Timaukel (Tierra del Fuego). Distribución en Chile: punta Patache (Iquique) a Magallanes (Quilhot et al. 2012). Distribución global: cosmopolita (Quilhot et al. 2012) (Exsiccatum: HIP:LIQ:15009).

Eurotiomycetes: Verrucariales

Verrucaciae

Normandina pulchella (Borrer) Nyl.

Hábitat: bosque, sobre el suelo y entre líquenes de bosque antiguo; ladera rocosa, con Pseudocyphellaria lechleri (Müll. Arg.) Du Rietz sobre superficies de rocas cerca de la orilla y asociado con Sticta hypochra Vain.; con Peltigera collina (Ach.) Schrad. sobre acantilado en pendiente a orientación sur. Registros en Magallanes: lago Pehoé, Parque Nacional Torres del Paine a 100 msnm (Última Esperanza); laguna Roca, Parque Nacional Torres del Paine a 250 msnm (Última Esperanza); refugio Pehoé, Parque Nacional Torres del Paine a 70 msnm (Última Esperanza). Distribución en Chile: archipiélago de Juan Fernández, y desde isla Mocha a Magallanes (Quilhot et al. 2012). Distribución global: islas Malvinas (Calvelo \& Fryday, 2006); cosmopolita (Quilhot et al. 2012) (Exsiccata: HIP:LIQ:15039; HIP:LIQ:15040; HIP:LIQ:15355).

Lecanoromycetes: Caliciales

Physciaceae

Phaeophyscia endococcinodes (Poelt) Essl.

Hábitat: bosque, sobre roca sombreada en bosque antiguo. Registros en Magallanes: laguna Roca, Parque Nacional Torres del Paine a 250 msnm (Última Esperanza). Distribución en Chile: Magallanes. Distribución global: pantropical con algunas extensiones en la región templada (Aptroot \& Sipman, 1991); reportes en Asia, África, Norteamérica, Sudamérica, Australia y Nueva Zelanda (Chesnokov \& Konoreva, 2015) (Exsiccatum: HIP:LIQ:15044).

Physconia perisidiosa (Erichsen) Moberg

Hábitat: ladera rocosa, entre briófitos y otros líquenes en pendiente rocosa protegida cerca del lago. Registros en Magallanes: lago Pehoé, Parque Nacional Torres del Paine a $70 \mathrm{msnm}$ (Última Esperanza). Distribución en Chile: Aysén a Magallanes (Quilhot et al. 2012). Distribución global: cosmopolita (Quilhot et al. 2012) (Exsiccatum: HIP:LIQ:15045). 
Lecanoromycetes: Lecanorales

Cladoniaceae

\section{Cladonia gracilis (L.) Willd.}

Hábitat: sin información disponible. Registros en Magallanes: cabecera del brazo norte del seno Hyatt, archipiélago de Tierra del Fuego (Magallanes). Distribución en Chile: Parque Katalapi (Pereira, 2007) y Magallanes. Distribución global: "cosmopolita, islas Orcadas del Sur, islas Shetland del Sur, península Antártica" (Redón, 1985) (Exsiccatum: HIP:LIQ:4478).

Cladonia gracilis (L.) Willd. subsp. elongata (Wulfen) Vain.

Hábitat: turbal esfagnoso; sobre troncos caídos en lugares muy húmedos y sombríos. Registros en Magallanes: aserradero Monte Alto, Rubens (Última Esperanza); fiordo Parry, bahía Cuevas a 100-200 msnm (Tierra del Fuego). Distribución en Chile: archipiélago de Juan Fernández, desde Osorno a Tierra del Fuego y Territorio Antártico (Quilhot et al. 2012). Distribución global: bipolar (Quilhot et al. 2012) (Exsiccata: HIP:LIQ:2914; HIP:LIQ:3706).

\section{Cladonia metacorallifera Asahina}

Hábitat: matorral, sobre el suelo denudado del matorral rastrero Empetrum; turbal esfagnoso; turbal, asociado con Polytrichum sobre rocas. Registros en Magallanes: bahía del Indio, lote San Isidro, río Yumbel (Magallanes); cabecera del brazo norte del seno Hyatt, archipiélago de Tierra del Fuego (Magallanes); embalse Lynch, Reserva Nacional Magallanes (Magallanes); fiordo Parry, bahía Cuevas a 100-200 msnm (Tierra del Fuego). Distribución en Chile: cordillera Pelada (Vergara \& Reyes, 2000), Reserva Nacional Tamango (Saucedo, 2004), Magallanes y Territorio Antártico (Redón, 1985). Distribución global: "América del Norte, Japón, Chile, Argentina y la Antártica; islas Orcadas del Sur, islas Shetland del Sur, península Antártica" (Redón, 1985) (Exsiccata: HIP:LIQ:2909; HIP:LIQ:2910; HIP:LIQ:4479; HIP:LIQ:8646).

\section{Cladonia mitis Sandst.}

Hábitat: bosque, lugares húmedos y sombríos del piso del bosque; turbal, turbal esfagnoso y común en turbales. Registros en Magallanes: laguna Parrillar, península de Brunswick (Magallanes); Penitente (Magallanes); fiordo Parry, bahía Cuevas a 100-200 msnm (Tierra del Fuego); surgidero Romanche, isla Otaries, archipiélago de Tierra del Fuego (Antártica). Distribución en Chile: provincia de Arauco a isla Navarino y Territorio Antártico (Quilhot et al. 2012). Distribución global: bipolar (Quilhot et al. 2012); "circumpolar en el hemisferio norte, extremo austral de América del Sur y península Antártica" (Redón, 1985) (Exsiccata: HIP:LIQ:2904; HIP:LIQ:3019; HIP:LIQ:4167; HIP:LIQ:8104).

\section{Cladonia pycnoclada (Pers.) Nyl.}

Hábitat: turbal, turbales esfagnosos y turbal alto interior, común en turbales; turbal esfagnoso; faldeos expuestos, sobre terrenos musgosos muy húmedos; terreno pantanoso. Registros en Magallanes: bahía Campana, isla Clarence, archipiélago de Tierra del Fuego (Magallanes); fiordo Silva Palma, angostura Titus (Magallanes); fondo de bahía Fortescue, estrecho de Magallanes (Magallanes); monte San Felipe, rinconada Bulnes a $490 \mathrm{msnm}$ (Magallanes); seno Resi, península Roca (Última Esperanza); aserradero Lago Blanco (Tierra del Fuego); caleta Martial, isla Herschel, archipiélago de Tierra del Fuego (Antártica); surgidero Romanche, isla Otaries, archipiélago de Tierra del Fuego (Antártica). Distribución en Chile: archipiélago de Juan Fernández, desde Curicó a Tierra del Fuego y Territorio Antártico (Quilhot et al. 2012). Distribución global: sur de Sudamérica y Tristán de Cunha (Quilhot et al. 2012) (Exsiccata: HIP:LIQ:2911; HIP:LIQ:2912; HIP:LIQ:3455; HIP:LIQ:3551; HIP:LIQ:3938; HIP:LIQ:3953; HIP:LIQ:4387; HIP:LIQ:8103; HIP:LIQ:8980).

\section{Cladonia rangiferina (L.) Weber ex F.H. Wigg.}

Hábitat: turbal, común en turbales. Registros en Magallanes: surgidero Romanche, isla Otaries, archipiélago de Tierra del Fuego (Antártica). Distribución en Chile: Temuco a Tierra del Fuego y Territorio Antártico (Quilhot et al. 2012). Distribución global: bipolar (Quilhot et al. 2012); "extremo austral de América del Sur y la Antártica. Islas Orcadas del Sur, islas Shetland del Sur, península Antártica" (Redón, 1985) (Exsiccatum: HIP:LIQ:14997). 
Cladonia rangiferina (L.) Weber ex F.H. Wigg. var. vicaria (R. Sant.) Ahti

Hábitat: turbal, forma céspedes en turbales. Registros en Magallanes: fiordo Parry, bahía Cuevas a 100-200 msnm (Tierra del Fuego). Distribución en Chile: Magallanes. Distribución global: "extremo austral de América del Sur y la Antártica; islas Orcadas del Sur, islas Shetland del Sur, península Antártica" (Redón, 1985) (Exsiccata: HIP:LIQ:2908; HIP:LIQ:2997).

Cladonia squamosa (Scop.) Hoffm. var. squamosa (Scop.) Hoffm.

Hábitat: bosque, cespitosa sobre el piso del bosque, y lugares húmedos y claros. Registros en Magallanes: fiordo Parry, bahía Cuevas a 100200 msnm (Tierra del Fuego). Distribución en Chile: Magallanes. Distribución global: bipolar y circumpolar en Hemisferio Norte (Lindsay, 1974) (Exsiccatum: HIP:LIQ:2905).

Cladonia subulata (L.) Weber ex F.H. Wigg.

Hábitat: turbal, turbal esfagnoso. Registros en Magallanes: laguna Parrillar, península de Brunswick (Magallanes). Distribución en Chile: Magallanes. Distribución global: ampliamente distribuida en Europa, también se conoce en África, Asia, Australia, Norteamérica y Sudamérica (Syrek \& Kukwa, 2008) (Exsiccatum: HIP:LIQ:4166).

\section{Haematommataceae}

\section{Haematomma nothofagi Kalb \& Staiger}

Hábitat: bosque, sobre corteza de Nothofagus pumilio (Poepp. \& Endl.) Krasser; sobre ramas y troncos de Nothofagus. Registros en Magallanes: camino a Club Andino, Reserva Nacional Magallanes (Magallanes); laguna Parrillar, península de Brunswick (Magallanes). Distribución en Chile: Magallanes. Distribución global: sureste de Australia, Nueva Zelanda y sur de Sudamérica (Australian Biological Resources Study, 2004) (Exsiccata: HIP:LIQ:4152; HIP:LIQ:8660).

\section{Haematomma puniceum (Ach.) A. Massal.}

Hábitat: bosque costero, sobre corteza de Nothofagus antarctica (G. Forst.) Oerst. Registros en Magallanes: mina Marta, seno Skyring (Magallanes). Distribución en Chile: Parque Nacional
Fray Jorge (Skottsberg, 1941), Magallanes e isla Navarino (Redón \& Quilhot, 1977). Distribución global: tropical y subtropical, zonas templadas frías en Australia y Nueva Zelanda, áreas alpinas de Nueva Zelanda (Rogers \& Hafellner, 1988) (Exsiccatum: HIP:LIQ:3361).

\section{Lecanoraceae}

Palicella glaucopa (Hook. f. \& Taylor) Rodr. Flakus \& Printzen

Hábitat: bosque, sobre corteza de Nothofagus pumilio. Registros en Magallanes: laguna Parrillar, península de Brunswick (Magallanes). Distribución en Chile: Araucanía a Magallanes (Rodríguez \& Printzen, 2014). Distribución global: sur de Sudamérica (Rodríguez \& Printzen, 2014) (Exsiccatum: HIP:LIQ:4157*).

\section{Parmeliaceae}

Alectoria ochroleuca (Schrank) A. Massal.

Hábitat: bajo matorral de Lepidophyllum cupressiforme (Lam.) Cass. Registros en Magallanes: punta Espora (Tierra del Fuego). Distribución en Chile: Llanquihue a isla Navarino (Quilhot et al. 2012). Distribución global: circumpolar (Quilhot et al. 2012) (Exsiccatum: HIP:LIQ:3728).

\section{Cetraria aculeata (Schreb.) Fr.}

Hábitat: pradera, exposición norte; sobre el suelo. Registros en Magallanes: lago Skottsberg, Parque Nacional Torres del Paine a $100 \mathrm{msnm}$ (Última Esperanza); laguna Azul, Parque Nacional Torres del Paine a 150 msnm (Última Esperanza). Distribución en Chile: Llanquihue a isla Navarino y Territorio Antártico (Quilhot et al. 2012). Distribución global: bipolar (Fernández-Mendoza \& Printzen, 2013); bipolar-alpino (Quilhot et al. 2012); cosmopolita (Randlane \& Saag, 2007) (Exsiccata: HIP:LIQ:15005; HIP:LIQ:15006).

Cetraria islandica (L.) Ach.

Hábitat: estepa, sobre el suelo en estepa anti-boreal con Empetrum. Registros en Magallanes: cañadón Bombalot, morro Chico a 100-150 msnm (Última Esperanza). Distribución en Chile: Magallanes (Redón \& Quilhot, 1977). 
Distribución global: bipolar (Redón \& Quilhot, 1977) (Exsiccatum: HIP:LIQ:15008).

Coelopogon epiphorellus (Nyl.) Brusse \& Kärnefelt

Hábitat: roca, sobre rocas en pendiente de exposición sur. Registros en Magallanes: lago Pehoé, Parque Nacional Torres del Paine a 100 msnm (Última Esperanza). Distribución en Chile: Parque Nacional La Campana, Talca a isla Navarino y Territorio Antártico (Quilhot et al. 2012). Distribución global: África-Sudamérica (Quilhot et al. 2012); sur de Sudamérica, Sudáfrica y región Antártica (Goffinet, 2012) (Exsiccatum: HIP:LIQ:15013).

\section{Hypogymnia lugubris (Pers.) Krog}

Hábitat: bosque, sobre corteza de Nothofagus pumilio y sobre ramas y troncos de Nothofagus. Registros en Magallanes: camino a Club Andino, Reserva Nacional Magallanes (Magallanes); laguna Parrillar, península de Brunswick (Magallanes). Distribución en Chile: Llanquihue a isla Navarino y Territorio Antártico (Quilhot et al. 2012). Distribución global: bipolar (Quilhot et al. 2012); "presente en Alaska, Asia, Australia, región austral de América del Sur y South Georgia", "siendo el primer hallazgo para la Región Antártica Marítima” (Redón, 1985) (Exsiccata: HIP:LIQ:4161*; HIP:LIQ:8662).

Hypogymnia lugubris (Pers.) Krog var. lugubris (Pers.) Krog

Hábitat: sin información disponible. Registros en Magallanes: Chabunco (Magallanes). Distribución en Chile: Magallanes. Distribución global: presente en Nueva Zelanda (de Lange et al. 2018), Australia (Schumm \& Elix, 2014) y extremo sur de Chile (Exsiccata: HIP:LIQ:3028; HIP:LIQ:15416*).

\section{Hypotrachyna brevirhiza (Kurok.) Hale}

Hábitat: bosque, sobre rocas sombreadas en bosque antiguo; lacustre, sobre superficie de roca húmeda cerca de lago. Registros en Magallanes: lago Pehoé, Parque Nacional Torres del Paine a 70 msnm (Última Esperanza); laguna Roca, Parque Nacional Torres del Paine a 250 msnm (Última Esperanza). Distribución en Chile: isla Mocha a Magallanes (Quilhot et al. 2012). Distribución global: pantropical y extendida en áreas temperadas de Argentina y Chile (Quilhot et al. 2012) (Exsiccata: HIP:LIQ:15015; HIP:LIQ:15016).

\section{Menegazzia cincinnata (Ach.) Bitter}

Hábitat: bosque, sobre corteza de Nothofagus pumilio. Registros en Magallanes: laguna Parrillar, península de Brunswick (Magallanes). Distribución en Chile: Termas de Chillán a isla Navarino (Rubio et al. 2013). Distribución global: endémica del sur de Sudamérica (Quilhot et al. 2012) (Exsiccatum: HIP:LIQ:15020).

Menegazzia globulifera R. Sant.

Hábitat: bosque, sobre corteza de Nothofagus pumilio. Registros en Magallanes: laguna Parrillar, península de Brunswick (Magallanes); aserradero Monte Alto, Rubens (Última Esperanza). Distribución en Chile: Termas de Chillán a isla Navarino (Rubio et al. 2013). Distribución global: austral (Quilhot et al. 2012); panaustral (Rubio et al. 2013) (Exsiccata: HIP:LIQ:3719*; HIP:LIQ:4155*; HIP:LIQ:4161*).

Menegazzia opuntioides (Müll. Arg.) R. Sant.

Hábitat: bosque, corticícola en Nothofagus pumilio. Registros en Magallanes: aserradero Monte Alto, Rubens (Última Esperanza). Distribución en Chile: Choshuenco a Magallanes (Quilhot et al. 2012). Distribución global: endémica del sur de Sudamérica (Quilhot et al. 2012) (Exsiccatum: HIP:LIQ:3719*).

Menegazzia sanguinascens (Räsänen) R. Sant.

Hábitat: abundante en sitios húmedos y sombríos; sobre corteza de Nothofagus. Registros en Magallanes: laguna Roca, Parque Nacional Torres del Paine a 250 msnm (Última Esperanza); río Bueno, Forestal Trillium, Timaukel (Tierra del Fuego). Distribución en Chile: Parque Nacional Nahuelbuta a isla Navarino (Quilhot et al. 2012). Distribución global: austral (Quilhot et al. 2012) (Exsiccata: HIP:LIQ:15024; HIP:LIQ:15033).

Menegazzia subpertusa P. James \& D. J. Galloway Hábitat: abundante en sitios húmedos y sombríos. Registros en Magallanes: río Bueno, Forestal Trillium, Timaukel (Tierra del Fuego). Distribución en Chile: Termas de Chillán (VIII 
Región) a Magallanes (Quilhot et al. 2012). Distribución global: austral (Quilhot et al. 2012) (Exsiccatum: HIP:LIQ:15025).

Menegazzia tenuis R. Sant.

Hábitat: bosque, sobre Nothofagus pumilio en bosque antiguo; sobre corteza de Nothofagus.

Registros en Magallanes: laguna Roca, Parque Nacional Torres del Paine a $250 \mathrm{msnm}$ (Última Esperanza). Distribución en Chile: Melipeuco e Icalma a Magallanes (Quilhot et al. 2012). Distribución global: endémica del sur de Sudamérica (Quilhot et al. 2012) (Exsiccata: HIP:LIQ:15026; HIP:LIQ:15380).

Menegazzia valdiviensis (Räsänen) R. Sant.

Hábitat: bosque, sobre corteza de Nothofagus pumilio. Registros en Magallanes: laguna Parrillar, península de Brunswick (Magallanes); cabecera del brazo norte del seno Hyatt, archipiélago de Tierra del Fuego (Magallanes). Distribución en Chile: Parque Nacional Nahuelbuta a isla Navarino (Quilhot et al. 2012). Distribución global: endémica del sur de Sudamérica (Quilhot et al. 2012) (Exsiccata: HIP:LIQ:4155*; HIP:LIQ:4393).

Nephromopsis chlorophylla (Willd.) Divakar, A. Crespo \& Lumbsch

Sinonimia: Tuckermanopsis chlorophylla (Willd.) Hale. Hábitat: bosque, sobre tronco muerto. Registros en Magallanes: puente del río Grey, Parque Nacional Torres del Paine a 50 msnm (Última Esperanza). Distribución en Chile: Parque Nacional La Campana a Magallanes (Quilhot et al. 2012). Distribución global: bipolar (Quilhot et al. 2012); común en ambos hemisferios, principalmente en bosques templados fríos $y$ restringida al medio oeste de la península Antártica (Passo et al. 2018) (Exsiccatum: HIP:LIQ:15007).

Notoparmelia protosulcata (Hale) A. Crespo, Ferencová \& Divakar

Sinonimia: Parmelia protosulcata Hale. Hábitat: ladera rocosa, sobre cara oeste de roca silícea. Registros en Magallanes: puente del río Grey, Parque Nacional Torres del Paine a 60 msnm (Última Esperanza). Distribución en Chile: Termas de Chillán a Magallanes (Quilhot et al.
2012). Distribución global: austral (Quilhot et al. 2012); Australia, Nueva Zelanda y América del Sur (Ferencová et al. 2014) (Exsiccatum: HIP:LIQ:15041).

Parmelia saxatilis (L.) Ach.

Hábitat: ladera rocosa, sobre cara oeste de roca silícea. Registros en Magallanes: puente del río Grey, Parque Nacional Torres del Paine a 60 msnm (Última Esperanza). Distribución en Chile: Parque Nacional La Campana, y desde provincia de Malleco a isla Navarino y Territorio Antártico (Quilhot et al. 2012). Distribución global: cosmopolita (Quilhot et al. 2012) (Exsiccatum: HIP:LIQ:15042).

\section{Parmotrema reticulatum (Taylor) M. Choisy}

Hábitat: bosque, sobre Nothofagus pumilio; sobre rocas en pendiente sur. Registros en Magallanes: lago Pehoé, Parque Nacional Torres del Paine a 100 msnm (Última Esperanza); laguna Roca, Parque Nacional Torres del Paine a 250 msnm (Última Esperanza). Distribución en Chile: Magallanes. Distribución global: reportada en todos los continentes, excepto Antártica (Divakar et al. 2005) (Exsiccata: HIP:LIQ:15374; HIP:LIQ:15375).

Platismatia glauca (L.) W.L. Culb. \& C.F. Culb. Hábitat: abundante en lugares húmedos. Registros en Magallanes: río Bueno, Forestal Trillium, Timaukel (Tierra del Fuego); isla Navarino, archipiélago de Tierra del Fuego (Antártica). Distribución en Chile: Parque Nacional La Campana, y desde Talca a isla Navarino y Territorio Antártico (Quilhot et al. 2012). Distribución global: islas Malvinas (Calvelo \& Fryday, 2006); cosmopolita (Quilhot et al. 2012) (Exsiccata: HIP:LIQ:3355; HIP:LIQ:15046).

\section{Protousnea dusenii (Du Rietz) Krog}

Hábitat: sobre tronco muerto de Nothofagus antarctica. Registros en Magallanes: laguna Larga, Parque Nacional Torres del Paine a $300 \mathrm{msnm}$ (Última Esperanza). Distribución en Chile: Aysén a isla Navarino (Quilhot et al. 2012). Distribución global: endémica del sur de Sudamérica (Quilhot et al. 2012) (Exsiccatum: HIP:LIQ:15048). 


\section{Protousnea magellanica (Mont.) Krog}

Hábitat: bosque, abundante en el bosque y corticícola en Nothofagus pumilio. Registros en Magallanes: aserradero Monte Alto, Rubens (Última Esperanza); río Bueno, Forestal Trillium, Timaukel (Tierra del Fuego). Distribución en Chile: Talca a isla Navarino (Quilhot et al. 2012). Distribución global: endémica del sur de Sudamérica (Quilhot et al. 2012) (Exsiccata: HIP:LIQ:3725; HIP:LIQ:15049).

\section{Punctelia reddenda (Stirt.) Krog}

Hábitat: acantilado empinado vertical de orientación norte. Registros en Magallanes: lago Grey, Parque Nacional Torres del Paine a 80 msnm (Última Esperanza). Distribución en Chile: precordillera altoandina de Arica y Parinacota (Herrera, 2009), isla Mocha (Gatica et al. 2011), Valdivia (Crespo et al. 2004). Distribución global: reportada en todos los continentes (Spielmann \& Marcelli, 2008) (Exsiccatum: HIP:LIQ:15376).

\section{Usnea aurantiacoatra (Jacq.) Bory}

Hábitat: bosque, en la línea del bosque sobre las rocas; turbal ciperoídeo, sobre rocas en sitios expuestos al viento; abundante en rocas desnudas; colectada en roca cerca de la cumbre de montaña. Registros en Magallanes: los Tres Morros (Magallanes); torre Triple a $1.219 \mathrm{msnm}$, cordillera Darwin (Tierra del Fuego); isla Hornos, archipiélago de Tierra del Fuego (Antártica); isla Wollaston, caleta Lientur a 300 msnm, archipiélago de Tierra del Fuego (Antártica). Distribución en Chile: Llanquihue a isla Navarino y Territorio Antártico (Quilhot et al. 2012). Distribución global: sur de Sudamérica y Antártica (Quilhot et al. 2012) (Exsiccata: HIP:LIQ:2897; HIP:LIQ:4382; HIP:LIQ:8813; HIP:LIQ:9022; HIP:LIQ:15036).

\section{Usnea igniaria Motyka}

Hábitat: bosque, epífito sobre troncos en sitios secos y luminosos. Registros en Magallanes: laguna Parrillar, península de Brunswick (Magallanes); cabecera del brazo norte del seno Hyatt, archipiélago de Tierra del Fuego (Magallanes). Distribución en Chile: Magallanes. Distribución global: islas Georgias del Sur y sur de Sudamérica (Lindsay, 1974) (Exsiccata: HIP:LIQ:2896; HIP:LIQ:4385).
Usnea trachycarpa (Stirt.) Müll. Arg.

Hábitat: escorial, en lugares expuestos sobre rocas volcánicas; desierto andino. Registros en Magallanes: escorial de Parque Nacional Pali Aike (Magallanes); laguna Timone (Magallanes); cerro Toro (Última Esperanza). Distribución en Chile: Llanquihue a isla Navarino y Territorio Antártico (Quilhot et al. 2012). Distribución global: endémica del sur de Sudamérica (Quilhot et al. 2012) (Exsiccata: HIP:LIQ:2895; HIP:LIQ:2898; HIP:LIQ:14996*).

\section{Xanthoparmelia hypopsila (Müll. Arg.) Hale}

Hábitat: sobre rocas expuestas al sol. Registros en Magallanes: laguna Amarga, Parque Nacional Torres del Paine (Última Esperanza). Distribución en Chile: Magallanes. Distribución global: Sudáfrica y Sudamérica (Estrabou \& Adler, 1999) (Exsiccatum: HIP:LIQ:15388).

\section{Xanthoparmelia microspora (Müll. Arg.) Hale}

Hábitat: sobre rocasexpuestasal sol. Registros en Magallanes: laguna Amarga, Parque Nacional Torres del Paine a 200 msnm (Última Esperanza). Distribución en Chile: Magallanes. Distribución global: endémica de Sudamérica (Elvebakk et al. 2014) (Exsiccatum: HIP:LIQ:15363).

Xanthoparmelia skottsbergiana T.H. Nash \& Elix Hábitat: sobre roca expuesta de forma intermedia densamente comprimida. Registros en Magallanes: lago Grey, Parque Nacional Torres del Paine a 70 msnm (Última Esperanza). Distribución en Chile: Magallanes. Distribución global: Sudamérica (Elvebakk et al. 2014) (Exsiccatum: HIP:LIQ:15362).

\section{Ramalinaceae}

Megalaria grossa (Pers. ex Nyl.) Hafellner

Hábitat: bosque, sobre corteza de Nothofagus pumilio. Registros en Magallanes: laguna Parrillar, península de Brunswick (Magallanes). Distribución en Chile: Maule a Magallanes (Quilhot et al. 1998). Distribución global: cosmopolita (Kantvilas, 2016) (Exsiccatum: HIP:LIQ:4157*).

Ramalina chilena (Nyl.) Kashiw.

Hábitat: epífito sobre matorral de Berberis buxifolia Lam. Registros en Magallanes: Pampa 
Alegre a 10 msnm, Punta Arenas (Magallanes). Distribución en Chile: isla Mocha (Quilhot et al. 2010) y Magallanes. Distribución global: "aparentemente endémica del sur de Sudamérica” (Quilhot et al. 2010) (Exsiccatum: HIP:LIQ:15407).

Ramalina farinacea (L.) Ach.

Hábitat: bosque, abundante en el bosque. Registros en Magallanes: río Bueno, Forestal Trillium, Timaukel (Tierra del Fuego). Distribución en Chile: Reserva Nacional Altos de Lircay (Pereira et al. 2014), isla Mocha (Quilhot et al. 2010) y Magallanes. Distribución global: circumpolar (Quilhot et al. 2010) (Exsiccata: HIP:LIQ:15400; HIP:LIQ:15401).

Ramalina terebrata Hook. f. \& Taylor

Hábitat: escorial, en lugares expuestos sobre rocas volcánicas; común sobre rocas en sitios protegidos por el viento; en zona de salpicaduras; saxícola y abundante. Registros en Magallanes: bahía Ventisquero, estrecho de Magallanes (Magallanes); escorial en Parque Nacional Pali Aike (Magallanes); laguna Timone (Magallanes); Parque Nacional Pali Aike a 200 msnm (Magallanes); cerro Los Onas, estancia Tres Arroyos, San Sebastián (Tierra del Fuego). Distribución en Chile: Magallanes, y en península Antártica e islas antárticas (Kappen et al. 1988). Distribución global: "regiones templadas y subantárticas de América del Sur, islas Orcadas del Sur, islas Shetland del Sur, península Antártica" (Redón, 1985) (Exsiccata: HIP:LIQ:2925; HIP:LIQ:3027; HIP:LIQ:3031; HIP:LIQ:4381; HIP:LIQ:12702; HIP:LIQ:14996*; HIP:LIQ:15406).

\section{Sphaerophoraceae}

Bunodophoron insigne (Laurer) Wedin

Hábitat: sobre Nothofagus betuloides (Mirb.) Oerst. en bosque de Drimys winteri J.R. Forst. \& G. Forst. Registros en Magallanes: península de Brunswick a 90 msnm (Magallanes). Distribución en Chile: Parque Nacional Nahuelbuta a Magallanes (Quilhot et al. 2012). Distribución global: Argentina, Chile, Nueva Zelanda, sur de Australia e islas Salomón (Quilhot et al. 2012) (Exsiccatum: HIP:LIQ:15000).
Bunodophoron patagonicum (C.W. Dodge) Wedin Hábitat: sobre Nothofagus betuloides en bosque de Drimys winteri. Registros en Magallanes: península de Brunswick a 90 msnm (Magallanes). Distribución en Chile: archipiélago de Juan Fernández y desde provincia de Malleco a Magallanes (Quilhot et al. 2012). Distribución global: austral (Quilhot et al. 2012) (Exsiccatum: HIP:LIQ:15002).

\section{Leifidium tenerum (Laurer) Wedin}

Hábitat: faldeos turbosos, muy húmedos en sitios expuestos; sobre corteza y ramillas de Berberis ilicifolia L.f. Registros en Magallanes: bahía Campana, isla Clarence, archipiélago de Tierra del Fuego (Magallanes); puerto Molyneux, isla Drummond Hay (Última Esperanza); isla Gordon, archipiélago de Tierra del Fuego (Antártica). Distribución en Chile: Valdivia a Magallanes (Quilhot et al. 2012). Distribución global: austral (Quilhot et al. 2012) (Exsiccata: HIP:LIQ:3460; HIP:LIQ:3472; HIP:LIQ:3954; HIP:LIQ:4330).

\section{Sphaerophorus globosus (Huds.) Vain.}

Hábitat: turbal, común en turbales. Registros en Magallanes: valle en Dientes de Navarino, isla Navarino a 525 msnm (Antártica); surgidero Romanche, isla Otaries, archipiélago de Tierra del Fuego (Antártica). Distribución en Chile: desde Llanquihue a isla Navarino y Territorio Antártico (Quilhot et al. 2012). Distribución global: bipolar (Quilhot et al. 2012) (Exsiccata: HIP:LIQ:15371; HIP:LIQ:15394).

\section{Stereocaulaceae}

\section{Stereocaulon alpinum Laurer}

Hábitat: sobre terrenos desvegetados arenoripiosos húmedos. Registros en Magallanes: fiordo Parry, glacial Reina Isabel II (Tierra del Fuego). Distribución en Chile: desde provincia de Cautín a isla Navarino y Territorio Antártico (Quilhot et al. 2012). Distribución global: bipolar (Quilhot et al. 2012) (Exsiccatum: HIP:LIQ:2900).

Stereocaulon caespitosum Redinger

Hábitat: colectado en arbustos. Registros en Magallanes: isla Tammar, estrecho de Magallanes (Magallanes). Distribución en Chile: Magallanes. 
Distribución global: Nueva Zelanda, Tasmania, Australia, e islas Georgias del Sur en la Antártica (Youn et al. 2018); sur de Sudamérica (Exsiccatum: HIP:LIQ:4390).

\section{Stereocaulon ramulosum Raeusch.}

Hábitat: morrena glacial; asociado con musgos, sobre rocas, en lugares altos y en semisombra; sobre rocas emergiendo de río correntoso. Registros en Magallanes: caleta Wilson, isla Clarence, archipiélago de Tierra del Fuego (Magallanes); laguna Parrillar, península de Brunswick (Magallanes); río Caleta (Magallanes); morrena del glaciar Upsala a 1.500 msnm, Hielo Patagónico Sur (Última Esperanza). Distribución en Chile: Talca a Magallanes y Territorio Antártico (Quilhot et al. 2012). Distribución global: cosmopolita (Quilhot et al. 2012) (Exsiccata: HIP:LIQ:2899; HIP:LIQ:3018; HIP:LIQ:3447; HIP:LIQ:7996).

Lecanoromycetes: Ostropales

Coenogoniaceae

\section{Coenogonium patagonicum Müll. Arg.}

Hábitat: epífito en lugares húmedos y claros (rojo cuando está fresco). Registros en Magallanes: bahía Transición, isla Capitán Aracena (Magallanes). Distribución en Chile: entre Zapallar y Cachagua (Redón, 1972), y Magallanes. Distribución global: sin información disponible (Exsiccatum: HIP:LIQ:2924).

Lecanoromycetes: Peltigerales

Lobariaceae

\section{Cyanisticta obvoluta (Sw.) C.W. Dodge}

Hábitat: bosque, sobre corteza de Nothofagus pumilio y sobre roca plana asociado con Nephroma spp. en bosque de viejo crecimiento de N. pumilio. Registros en Magallanes: sendero Zapata, Parque Nacional Torres del Paine a 200 msnm (Última Esperanza); aserradero Lago Blanco (Tierra del Fuego); isla Navarino, archipiélago de Tierra del Fuego (Antártica). Distribución en Chile: Parque Nacional Nahuelbuta a isla Navarino (Quilhot et al. 2012). Distribución global: endémica del sur de Sudamérica (Quilhot et al. 2012) (Exsiccata: HIP:LIQ:3016; HIP:LIQ:3356*; HIP:LIQ:15408).
Podostictina berberina (G. Forst.) Moncada \& Lücking

Sinonimia: Pseudocyphellaria berberina (G. Forst.) D.J. Galloway \& P. James. Hábitat: bosque, sobre troncos en sitios húmedos $y$ algo claros, en bosque profundo, epífito sobre Nothofagus betuloides en semisombra y sobre $N$. betuloides en sitios claros; epífito sobre troncos en lugares húmedos y sombríos; epífito sobre troncos caídos en sitios algo claros; sobre frondas secas de Blechnum magellanicum Mett. en sitios abiertos; corticícola en sitios húmedos; matorral enano turboso; turbal, abundante con Empetrum; turbal ciperoídeo, sobre superficie del turbal. Registros en Magallanes: brazo norte de seno Hyatt, archipiélago de Tierra del Fuego (Magallanes); fiordo Silva Palma, angostura Titus (Magallanes); isla Tammar, estrecho de Magallanes (Magallanes); puerto Henry, isla Riesco (Magallanes); río Caleta (Magallanes); fiordo Taraba, Spire fjord (Última Esperanza); puerto Molyneux, isla Drummond Hay (Última Esperanza); seno Resi, península Roca (Última Esperanza); bahía Agua Dulce, isla Clarence, archipiélago de Tierra del Fuego (Antártica); bahía Campana, isla Clarence, archipiélago de Tierra del Fuego (Antártica); caleta Martial, isla Herschel, archipiélago de Tierra del Fuego (Antártica); isla Hornos, archipiélago de Tierra del Fuego (Antártica); surgidero Romanche, isla Otaries, archipiélago de Tierra del Fuego (Antártica); seno Darwin, isla Burnt, archipiélago de Tierra del Fuego (Antártica). Distribución en Chile: archipiélago de Juan Fernández, y desde isla Mocha a Tierra del Fuego (Quilhot et al. 2012). Distribución global: endémica del sur de Sudamérica (Quilhot et al. 2012) (Exsiccata: HIP:LIQ:2919; HIP:LIQ:2920; HIP:LIQ:2921; HIP:LIQ:2923; HIP:LIQ:3473; HIP:LIQ:3474; HIP:LIQ:3889; HIP:LIQ:3922; HIP:LIQ:3939; HIP:LIQ:4323; HIP:LIQ:4386; HIP:LIQ:4396; HIP:LIQ:8106*; HIP:LIQ:8978; HIP:LIQ:14985; HIP:LIQ:14988*; HIP:LIQ:14991).

Podostictina endochrysa (Delise) D.J. Galloway \& de Lange

Sinonimia: Pseudocyphellaria endochrysa (Delise) Vain. Hábitat: bosque, escaso sobre piso del bosque decíduo; estepa, sobre piso de estepa asociado con Pseudocyphellaria glabra (Delise) Malme y Empetrum; epífito sobre 
troncos caídos en sitios algo claros. Registros en Magallanes: Chabunco (Magallanes); fiordo Silva Palma, angostura Titus (Magallanes); aserradero Monte Alto, Rubens (Última Esperanza); cañadón Bombalot, morro Chico a 100-150 msnm (Última Esperanza); isla Navarino, archipiélago de Tierra del Fuego (Antártica). Distribución en Chile: Aysén a isla Navarino (Quilhot et al. 2012). Distribución global: endémica del sur de Sudamérica (Quilhot et al. 2012) (Exsiccata: HIP:LIQ:3023; HIP:LIQ:3356*; HIP:LIQ:3690; HIP:LIQ:14990; HIP:LIQ:15051*; HIP:LIQ:15052).

\section{Podostictina scabrosa (R. Sant.) D.J. Galloway \& de Lange}

Sinonimia: Pseudocyphellaria scabrosa R. Sant. Hábitat: bosque, muy escaso en corteza de árboles en sitios húmedos y escaso en bosque mixto ocupando como sustrato ramas de Nothofagus betuloides en descomposición; en ramita seca en pendiente sur. Registros en Magallanes: lago Pehoé, Parque Nacional Torres del Paine a 100 msnm (Última Esperanza); río Bueno, Forestal Trillium, Timaukel (Tierra del Fuego); isla Navarino, archipiélago de Tierra del Fuego (Antártica). Distribución en Chile: isla Mocha a isla Navarino (Quilhot et al. 2012). Distribución global: endémica del sur de Sudamérica (Quilhot et al. 2012). (Exsiccata: HIP:LIQ:14978*; HIP:LIQ:14994; HIP:LIQ:15063; HIP:LIQ:15379).

Podostictina vaccina (Mont.) D.J. Galloway \& de Lange

Sinonimia: Pseudocyphellaria vaccina (Mont.) Malme. Hábitat: bosque, sobre el suelo de bosque antiguo; sobre el suelo en la punta de una colina. Registros en Magallanes: Chorrillo de Salmones Parque Nacional Torres del Paine a 110 msnm (Última Esperanza); valle Asencio, Parque Nacional Torres del Paine a 500 msnm (Última Esperanza). Distribución en Chile: isla Mocha a isla Navarino (Quilhot et al. 2012). Distribución global: endémica del sur de Sudamérica (Quilhot et al. 2012) (Exsiccata: HIP:LIQ:15383; HIP:LIQ:15409).

Pseudocyphellaria coriifolia (Müll. Arg.) Malme Hábitat: bosque, corticícola en Nothofagus pumilio, escaso en bosque de $N$. pumilio $y$ escaso en sitios húmedos del bosque. Registros en Magallanes: aserradero Monte Alto, Rubens (Última Esperanza); río Bueno, Forestal Trillium, Timaukel (Tierra del Fuego); isla Navarino, archipiélago de Tierra del Fuego (Antártica). Distribución en Chile: archipiélago de Juan Fernández, y desde Chillán a isla Navarino (Quilhot et al. 2012). Distribución global: endémica del sur de Sudamérica (Quilhot et al. 2012) (Exsiccata: HIP:LIQ:3357*; HIP:LIQ:3721; HIP:LIQ:14981*; HIP:LIQ:14993; HIP:LIQ:15010*).

Pseudocyphellaria crocata (L.) Vain.

Hábitat: bosque, epífito sobre troncos caídos en sitios algo claros del bosque y escaso en bosque de N. pumilio; sobre el suelo en asociación con Pseudocyphellaria lechleri. Registros en Magallanes: fiordo Silva Palma, angostura Titus (Magallanes); laguna Roca, Parque Nacional Torres del Paine a 250 msnm (Última Esperanza); río Bueno, Forestal Trillium, Timaukel (Tierra del Fuego); isla Navarino, archipiélago de Tierra del Fuego (Antártica). Distribución en Chile: archipiélago de Juan Fernández, Parque Nacional Fray Jorge, y desde Talca a isla Navarino (Quilhot et al. 2012). Distribución global: cosmopolita (Quilhot et al. 2012) (Exsiccata: HIP:LIQ:2922; HIP:LIQ:3353; HIP:LIQ:3358*; HIP:LIQ:14978*; HIP:LIQ:14981*; HIP:LIQ:14983; HIP:LIQ:15010*; HIP:LIQ:15034).

Pseudocyphellaria divulsa (Taylor) Imshaug Hábitat: sin información disponible. Registros en Magallanes: puerto Molyneux, isla Drummond Hay (Última Esperanza). Distribución en Chile: provincia de Malleco a Magallanes (Quilhot et al. 2012). Distribución global: endémica del sur de Sudamérica (Quilhot et al. 2012) (Exsiccatum: HIP:LIQ:4324).

\section{Pseudocyphellaria dubia Du Rietz}

Hábitat: bosque, sobre tocón de Nothofagus betuloides en bosque antiguo y sobre $N$. betuloides en bosque de viejo crecimiento, expuesto a muy intensa herbivoría por arañas; en tronco de $N$. betuloides asociado con Sticta hypochra y Pseudocyphellaria crocata (L.) Vain. Registros en Magallanes: cascada Pingo, Parque Nacional Torres del Paine a 170 m y 180 msnm (Última Esperanza); refugio Zapata, Parque Nacional 
Torres del Paine a 180 msnm (Última Esperanza); valle Asencio, Parque Nacional Torres del Paine a 500 msnm (Última Esperanza). Distribución en Chile: provincia de Malleco a isla Navarino (Quilhot et al. 2012). Distribución global: endémica del sur de Sudamérica (Quilhot et al. 2012) (Exsiccata: HIP:LIQ:15038; HIP:LIQ:15047; HIP:LIQ:15050; HIP:LIQ:15390).

Pseudocyphellaria faveolata (Delise) Malme

Hábitat: bosque, epífito sobre Nothofagus betuloides en semisombra y en bosque mixto de $N$. pumilio y $N$. betuloides abundante en lugares húmedos y sombríos; roca, entre musgos sobre rocas. Registros en Magallanes: río Caleta (Magallanes); lago Skottsberg, Parque Nacional Torres del Paine a 100 msnm (Última Esperanza); río Bueno, Forestal Trillium, Timaukel (Tierra del Fuego); isla Navarino, archipiélago de Tierra del Fuego (Antártica). Distribución en Chile: Parque Nacional Nahuelbuta a Tierra del Fuego (Quilhot et al. 2012). Distribución global: panaustral; también en islas subantárticas, Nueva Zelanda y Tasmania (Quilhot et al. 2012) (Exsiccata: HIP:LIQ:2916; HIP:LIQ:3357*; HIP:LIQ:14982; HIP:LIQ:15054; HIP:LIQ:15059).

Pseudocyphellaria glabra (Hook. f. \& Taylor) C.W. Dodge

Hábitat: bosque litoral, en lugares claros y húmedos del piso del bosque; turbal, abundante en turbales con Empetrum y en turbera de Sphagnum sobre Empetrum; turbal ciperoídeo, sobre superficie del turbal; matorral, sobre el suelo entre matorrales enanos con Gaultheria mucronata (L.f.) Hook. \& Arn. y Empetrum; epifito sobre troncos caídos en sitios algo claros; sobre el piso en cresta expuesta; abundante en lugares húmedos. Registros en Magallanes: fiordo Silva Palma, angostura Titus (Magallanes); laguna Parrillar, península de Brunswick (Magallanes); Reserva Nacional Magallanes (Magallanes); cañadón Bombalot, morro Chico a 100-150 msnm (Última Esperanza); lago Skottsberg, Parque Nacional Torres del Paine a 100 msnm (Última Esperanza); morrena en valle Asencio, Parque Nacional Torres del Paine a 900 msnm (Última Esperanza); río Bueno, Forestal Trillium, Timaukel (Tierra del Fuego); fiordo Parry, bahía Cuevas (Tierra del Fuego); surgidero Romanche, isla
Otaries, archipiélago de Tierra del Fuego (Antártica); isla Hornos, archipiélago de Tierra del Fuego (Antártica). Distribución en Chile: archipiélago de Juan Fernández, isla Mocha a Tierra del Fuego (Quilhot et al. 2012). Distribución global: panaustral (Quilhot et al. 2012) (Exsiccata: HIP:LIQ:2917; HIP:LIQ:2918; HIP:LIQ:3014; HIP:LIQ:8106*; HIP:LIQ:8659; HIP:LIQ:14988*; HIP:LIQ:15051*; HIP:LIQ:15055; HIP:LIQ:15056; HIP:LIQ:15057; HIP:LIQ:15058; HIP:LIQ:15062; HIP:LIQ:15359).

\section{Pseudocyphellaria granulata (C. Bab.) Malme \\ Hábitat: bosque, corticícola en Nothofagus pumilio. Registros en Magallanes: laguna Parrillar, península de Brunswick (Magallanes); aserradero Monte Alto, Rubens (Última Esperanza). Distribución en Chile: Parque Nacional Nahuelbuta a isla Navarino (Quilhot et al. 2012). Distribución global: austral (Quilhot et al. 2012) (Exsiccata: HIP:LIQ:3724; HIP:LIQ:4151).}

Pseudocyphellaria hirsuta (Mont.) Malme

Hábitat: bosque, sobre briófitos en el suelo en bosque de viejo crecimiento; colectado en árbol. Registros en Magallanes: cabecera del brazo norte del seno Hyatt, archipiélago de Tierra del Fuego (Magallanes); laguna Roca, Parque Nacional Torres del Paine a $250 \mathrm{msnm}$ (Última Esperanza); isla Navarino, archipiélago de Tierra del Fuego (Antártica).

Distribución en Chile: archipiélago de Juan Fernández, y desde Constitución a Tierra del Fuego (Quilhot et al. 2012). Distribución global: endémica del sur de Sudamérica (Quilhot et al. 2012). (Exsiccata: HIP:LIQ:3352; HIP:LIQ:4384; HIP:LIQ:15393).

Pseudocyphellaria intricata (Delise) Vain.

Hábitat: bosque, en rocas sombreadas en bosque antiguo con pseudocyphellae indistinta, pero no se observó cyphellae; en cara norte de rocas cerca de la orilla. Registros en Magallanes: laguna Roca, Parque Nacional Torres del Paine a 250 msnm (Última Esperanza); refugio Pehoé, Parque Nacional Torres del Paine a 70 msnm (Última Esperanza). Distribución en Chile: archipiélago de Juan Fernández, Parque Nacional Fray Jorge a Tierra del Fuego (Quilhot et al. 2012). Distribución 
global: cosmopolita (Quilhot et al. 2012)(Exsiccata: HIP:LIQ:15381; HIP:LIQ:15382).

Pseudocyphellaria lechleri (Müll. Arg.) Du Rietz

Hábitat: bosque, sobre corteza de Nothofagus pumilio, sobre el suelo asociado con Pseudocyphellaria crocata y abundante pseudocyphellae, ambos a lo largo de márgenes en lado superior y abundante en lugares húmedos del bosque; matorral enano, en suelo entre musgos y matorrales enanos; bajo matorral de Lepidophyllum cupressiforme; sobre el suelo en pendiente sur; sobre cara norte de rocas cerca de la orilla. Registros en Magallanes: laguna Parrillar, península de Brunswick (Magallanes); lago Skottsberg, Parque Nacional Torres del Paine a 100 msnm (Última Esperanza); laguna Roca, Parque Nacional Torres del Paine a 250 msnm (Última Esperanza); refugio Paine, Parque Nacional Torres del Paine a 100 msnm (Última Esperanza); refugio Pehoé, Parque Nacional Torres del Paine a 70 msnm (Última Esperanza); aserradero Lago Blanco (Tierra del Fuego); punta Espora (Tierra del Fuego); río Bueno, Forestal Trillium, Timaukel (Tierra del Fuego); isla Navarino, archipiélago de Tierra del Fuego (Antártica). Distribución en Chile: Parque Nacional Conguillío a Tierra del Fuego (Quilhot et al. 2012). Distribución global: endémica del sur de Sudamérica (Quilhot et al. 2012) (Exsiccata: HIP:LIQ:3017; HIP:LIQ:3020; HIP:LIQ:3358*; HIP:LIQ:3729; HIP:LIQ:4164; HIP:LIQ:14981*; HIP:LIQ:15030;HIP:LIQ:15064;HIP:LIQ:15353; HIP:LIQ:15378; HIP:LIQ:15384).

\section{Sticta caulescens De Not.}

Hábitat: bosque, sobre Nothofagus pumilio cerca del río y sobre madera muerta en sitios claros; bosque costero; epífito sobre troncos, lugares húmedos y sombríos. Registros en Magallanes: puerto Henry, isla Riesco (Magallanes); fiordo Taraba, Spire fjord (Última Esperanza); lago Grey, Parque Nacional Torres del Paine a 60 msnm (Última Esperanza); seno Resi, península Roca (Última Esperanza). Distribución en Chile: isla Mocha a Magallanes (Quilhot et al. 2012). Distribución global: endémica del sur de Sudamérica (Quilhot et al. 2012) (Exsiccata: HIP:LIQ:2903; HIP:LIQ:8981; HIP:LIQ:15350; HIP:LIQ:15405).
Sticta fuliginosa (Dicks.) Ach.

Hábitat: sobre rocas orientadas al norte cerca del mar asociado con Pseudocyphellaria lechleri y Pseudocyphellaria intricata (Delise) Vain.; sobre el suelo con otros líquenes foliosos en pendiente pronunciada. Registros en Magallanes: península de Brunswick a 40 msnm (Magallanes); refugio Pehoé, Parque Nacional Torres del Paine a 70 msnm (Última Esperanza). Distribución en Chile: Putre y desde Parque Nacional Fray Jorge a Magallanes (Quilhot et al. 2012). Distribución global: cosmopolita (Quilhot et al. 2012) (Exsiccata: HIP:LIQ:15395; HIP:LIQ:15404).

\section{Sticta hypochra Vain.}

Hábitat: bosque, entre musgos sobre rocas en bosque antiguo sin estípites distintivos y en roca sombreada en bosque antiguo; sobre el suelo con musgos y otros líquenes foliosos en pendiente empinada; especímenes muy pequeños que crecen directamente sobre rocas en pendiente de orientación sur; abundante en pendientes cerca de ríos. Registros en Magallanes: península de Brunswick a $40 \mathrm{msnm}$ (Magallanes); río Bueno, Forestal Trillium, Timaukel (Tierra del Fuego); lago Pehoé, Parque Nacional Torres del Paine a 100 msnm (Última Esperanza); laguna Roca, Parque Nacional Torres del Paine a 250 msnm (Última Esperanza); isla Navarino, archipiélago de Tierra del Fuego (Antártica). Distribución en Chile: desde el Parque Nacional Conguillio a isla Navarino (Quilhot et al. 2012). Distribución global: endémica del sur de Sudamérica (Quilhot et al. 2012) (Exsiccata: HIP:LIQ:14978*; HIP:LIQ:15351; HIP:LIQ:15370; HIP:LIQ:15392; HIP:LIQ:15397; HIP:LIQ:15398).

\section{Sticta limbata (Sm.) Ach.}

Hábitat: sobre el suelo o en ramitas viejas secas del suelo; en pendiente de orientación sur; sobre el suelo en superficie de roca vertical pequeña orientada al sur. Registros en Magallanes: lago Pehoé, Parque Nacional Torres del Paine a $80 \mathrm{~m}$ y $100 \mathrm{msnm}$ (Última Esperanza). Distribución en Chile: isla Mocha a Tierra del Fuego (Quilhot et al. 2012). Distribución global: cosmopolita (Quilhot et al. 2012) (Exsiccata: HIP:LIQ:15356; HIP:LIQ:15387).

\section{Sticta santessonii D.J. Galloway}

Hábitat: sobre rocas entre Racomitrium lanuginosum (Hedw.) Brid. cerca de un afluente 
de río. Registros en Magallanes: lago Grey, Parque Nacional Torres del Paine a $80 \mathrm{msnm}$ (Última Esperanza). Distribución en Chile: Parque Nacional Conguillío a Magallanes (Quilhot et al. 2012). Distribución global: endémica del sur de Sudamérica (Quilhot et al. 2012) (Exsiccatum: HIP:LIQ:15369).

\section{Sticta weigelii (Ach.) Vain.}

Hábitat: bosque, sobre afloramiento de roca orientada al norte de bosque viejo de Nothofagus betuloides y sobre el suelo de bosque abierto; sobre el suelo con briófitas y líquenes crustosos en cara sur de la pendiente, mayormente filidios; sobre el suelo asociado con Pseudocyphellaria freycinetii y $P$. lechleri; sobre roca cubierta de musgos y líquenes foliosos; entre briófitas en rocas cerca del lago. Registros en Magallanes: península de Brunswick a 40 msnm (Magallanes); lago Pehoé, Parque Nacional Torres del Paine a 100 msnm (Última Esperanza); lago Skottsberg, Parque Nacional Torres del Paine a 70 m, 75 m y 80 msnm (Última Esperanza); laguna Roca, Parque Nacional Torres del Paine a 250 msnm (Última Esperanza); refugio Zapata, Parque Nacional Torres del Paine a $180 \mathrm{msnm}$ (Última Esperanza). Distribución en Chile: Parque Nacional Fray Jorge y desde Parque Nacional Conguillío a isla Navarino (Quilhot et al. 2012). Distribución global: paleotropical (Quilhot et al. 2012) (Exsiccata: HIP:LIQ:15352; HIP:LIQ:15357; HIP:LIQ:15364; HIP:LIQ:15366; HIP:LIQ:15367; HIP:LIQ:15368; HIP:LIQ:15391; HIP:LIQ:15396).

\section{Nephromataceae}

Nephroma antarcticum (Wulfen) Nyl. var. lobuligerum Müll. Arg.

Hábitat: bosque, sobre tronco de Nothofagus antarctica en bosque antiguo; roca, sobre roca plana asociada a Pseudocyphellaria obvoluta (Sw.) Malme. Registros en Magallanes: laguna Parrillar, península de Brunswick (Magallanes); mina Loreto (Magallanes); refugio Zapata, Parque Nacional Torres del Paine (Última Esperanza); refugio Pingo, Parque Nacional Torres del Paine a 200 msnm (Última Esperanza). Distribución en Chile: archipiélago de Juan Fernández, desde provincia de Malleco a Magallanes (Quilhot et al. 2012). Distribución global: endémica del sur de Sudamérica (Quilhot et al. 2012) (Exsiccata: HIP:LIQ:3026; HIP:LIQ:3030; HIP:LIQ:15029; HIP:LIQ:15358).

Nephroma australe A. Rich.

Hábitat: bosque, epífito sobre Nothofagus betuloides en semisombra, sobre corteza y troncos de N. pumilio y abundante en sitios húmedos del bosque; corticícola en $N$. pumilio; sobre troncos caídos en sitios húmedos y algo claros; corticícola en sitios húmedos. Registros en Magallanes: bahía Agua Dulce, isla Clarence, archipiélago de Tierra del Fuego (Magallanes); cabecera del brazo norte del seno Hyatt, archipiélago de Tierra del Fuego (Magallanes); fiordo Silva Palma, angostura Titus (Magallanes); laguna Parrillar, península de Brunswick (Magallanes); río Caleta (Magallanes); aserradero Monte Alto, Rubens (Última Esperanza); aserradero Lago Blanco (Tierra del Fuego); río Bueno, Forestal Trillium, Timaukel (Tierra del Fuego). Distribución en Chile: archipiélago de Juan Fernández, y desde Llanquihue a Magallanes (Quilhot et al. 2012). Distribución global: austral (Quilhot et al. 2012) (Exsiccata: HIP:LIQ:2901; HIP:LIQ:2902; HIP:LIQ:3013; HIP:LIQ:3723; HIP:LIQ:3449; HIP:LIQ:3553; HIP:LIQ:4163; HIP:LIQ:4397; HIP:LIQ:15031; HIP:LIQ:15032).

\section{Nephroma cellulosum (Sm.) Ach.}

Hábitat: bosque, sobre tronco muerto de Nothofagus antarctica expuesto al sol. Registros en Magallanes: refugio Zapata, Parque Nacional Torres del Paine a 100 msnm (Última Esperanza). Distribución en Chile: archipiélago de Juan Fernández, y desde Concepción a isla Navarino (Quilhot et al. 2012). Distribución global: austral (Quilhot et al. 2012) (Exsiccatum: HIP:LIQ:15035).

\section{Nephroma papillosum F.J. White \& P. James}

Hábitat: sin información disponible.

Registros en Magallanes: mina Loreto (Magallanes).

Distribución en Chile: Parque Nacional Puyehue, volcán Osorno y Magallanes (White \& James, 1988). Distribución global: restringida a zonas altas de Chile y Argentina, y zonas bajas en la Patagonia (White \& James, 1988) (Exsiccatum: HIP:LIQ:15027). 


\section{Pannariaceae}

Pannaria athroophylla (Stirt.) Elvebakk \& D.J. Galloway

Sinonimia: Psoroma athroophyllum Stirt. Hábitat: sobre Nothofagus pumilio asociado con otras especies de Psoroma y Nephroma antarcticum (Wulfen) Nyl. Registros en Magallanes: refugio Pingo, Parque Nacional Torres del Paine a 110 msnm (Última Esperanza); isla Navarino, archipiélago de Tierra del Fuego (Antártica). Distribución en Chile: Magallanes. Distribución global: endémica de Nueva Zelanda (Elvebakk \& Galloway, 2003); Sudamérica (Passo \& Calvelo, 2011) (Exsiccata: HIP:LIQ:3350*; HIP:LIQ:14998*; HIP:LIQ:15389*).

Pannaria calophylla (Müll. Arg.) Passo \& Calvelo

Hábitat: corticícola en Nothofagus pumilio; epífito sobre troncos caídos en sitios algo claros. Registros en Magallanes: fiordo Silva Palma, angostura Titus (Magallanes); aserradero Monte Alto, Rubens (Última Esperanza). Distribución en Chile: desde Valdivia a Aysén (Quilhot et al. 2012). Distribución global: austral (Quilhot et al. 2012) (Exsiccata: HIP:LIQ:3720; HIP:LIQ:3722; HIP:LIQ:15051*).

Pannaria contorta (Müll. Arg.) Passo \& Calvelo

Hábitat: bosque, sobre corteza de Nothofagus pumilio. Registros en Magallanes: laguna Parrillar, península de Brunswick (Magallanes); isla Navarino, archipiélago de Tierra del Fuego (Antártica). Distribución en Chile: Tierra del Fuego y fiordo Andrew (Passo \& Calvelo, 2006). Distribución global: Nueva Zelanda, sur de Sudamérica (Passo \& Calvelo, 2006) (Exsiccata: HIP:LIQ:4158; HIP:LIQ:4162*; HIP:LIQ:14989*; HIP:LIQ:14998*).

Pannaria farinosa Elvebakk \& Fritt-Rasm.

Hábitat: bosque, sobre roca sombreada en bosque antiguo y sobre Nothofagus antarctica en bosque anegadizo asociado con Psoroma de mayor tamaño; sobre Nothofagus pumilio asociado con otras especies de Psoroma y Nephroma antarcticum. Registros en Magallanes: cerro Donoso, Parque Nacional Torres del Paine a 50 msnm (Última Esperanza); laguna Roca, Parque Nacional Torres del Paine a 250 msnm (Última
Esperanza); refugio Pingo, Parque Nacional Torres del Paine a 110 msnm (Última Esperanza); isla Navarino, archipiélago de Tierra del Fuego (Antártica). Distribución en Chile: archipiélago de Juan Fernández, Parque Nacional Fray Jorge, y desde Talca a isla Navarino (Quilhot et al. 2012). Distribución global: panaustral (Quilhot et al. 2012) (Exsiccata: HIP:LIQ:3350*; HIP:LIQ:15360; HIP:LIQ:15377; HIP:LIQ:15389*).

\section{Pannaria leproloma (Nyl.) P.M. Jørg.}

Hábitat: bosque, sobre Drimys winteri en sitios claros. Registros en Magallanes: seno Resi, península Roca (Última Esperanza). Distribución en Chile: Magallanes. Distribución global: Australia, endémica de Nueva Zelanda (Elvebakk et al. 2007) (Exsiccatum: HIP:LIQ:8979).

\section{Pannaria pallida (Nyl.) Hue}

Hábitat: bosque, escaso en sitios húmedos del bosque, sobre la corteza de Nothofagus pumilio y en sitios húmedos presente en corteza de árboles podridos. Registros en Magallanes: laguna Parrillar, península de Brunswick (Magallanes); valle Francés, Parque Nacional Torres del Paine (Última Esperanza); río Bueno, Forestal Trillium, Timaukel (Tierra del Fuego). Distribución en Chile: Talca a isla Navarino (Quilhot et al. 2012). Distribución global: austral (Quilhot et al. 2012) (Exsiccata: HIP:LIQ:14989*; HIP:LIQ:15372; HIP:LIQ:15385; HIP:LIQ:15399*; HIP:LIQ:15402*).

Pannaria patagonica (Malme) Elvebakk \& D. J. Galloway

Hábitat: bosque, escaso en sitios húmedos del bosque, sobre corteza de Nothofagus pumilio, presente en corteza de árboles podridos en sitios húmedos y sobre roca sombreada en bosque antiguo. Registros en Magallanes: laguna Parrillar, península de Brunswick (Magallanes); laguna Roca, Parque Nacional Torres del Paine a $250 \mathrm{msnm}$ (Última Esperanza); río Bueno, Forestal Trillium, Timaukel (Tierra del Fuego). Distribución en Chile: Tierra del Fuego y península de Brunswick (Sehnal, 2012). Distribución global: Nueva Zelanda y sur de Sudamérica (Elvebakk \& Galloway, 2003) (Exsiccata: HIP:LIQ:4159; HIP:LIQ:4162*; HIP:LIQ:4165; HIP:LIQ:15361; HIP:LIQ:15399*; HIP:LIQ:15402*; HIP:LIQ:15403). 
Siphulastrum mamillatum (Hook. f. \& Taylor) D.J. Galloway

Hábitat: zona de salpicaduras. Registros en Magallanes: brazo norte del seno Hyatt, archipiélago de Tierra del Fuego (Magallanes). Distribución en Chile: Magallanes. Distribución global: circumantártica (Øvstedal \& Gremmen, 2006) (Exsiccatum: HIP:LIQ:4391).

Xanthopsoroma contextum (Stirt.) Elvebakk

Hábitat: bosque, sobre corteza de Nothofagus pumilio. Registros en Magallanes: laguna Parrillar, península de Brunswick (Magallanes). Distribución en Chile: archipiélago de Juan Fernández, y desde provincia de Malleco a Magallanes (Quilhot et al. 2012). Distribución global: austral (Quilhot et al. 2012) (Exsiccatum: HIP:LIQ:4160).

Xanthopsoroma soccatum (R. Br. ex Cromb.) Elvebakk

Hábitat: colectado en árbol. Registros en Magallanes: cabecera del brazo norte del seno Hyatt, archipiélago de Tierra del Fuego (Magallanes). Distribución en Chile: archipiélago de Juan Fernández y desde Villarrica a Aysén (Quilhot et al. 2012). Distribución global: australasia (Quilhot et al. 2012) (Exsiccatum: HIP:LIQ:4392).

\section{Peltigeraceae}

Peltigera didactyla (With.) J.R. Laundon

Hábitat: sobre el suelo en fisuras de rocas cerca del lago. Registros en Magallanes: lago Pehoé, Parque Nacional Torres del Paine a 70 msnm (Última Esperanza). Distribución en Chile: Zapahuira-Putre (3400 m altitud), Parque Nacional La Campana, y desde Los Queñes a Aysén y Territorio Antártico (Quilhot et al. 2012). Distribución global: cosmopolita (Quilhot et al. 2012) (Exsiccatum: HIP:LIQ:15354). ,Peltigera polydactylon (Neck.) Hoffm.

Hábitat: bosque, epifito sobre troncos caídos en sitios algo claros del bosque. Registros en Magallanes: fiordo Silva Palma, angostura Titus (Magallanes). Distribución en Chile: Parque Nacional Fray Jorge (Skottsberg, 1941), Parque Nacional La Campana, y desde Aysén a isla Navarino (Quilhot et al. 2012). Distribución global: cosmopolita (Quilhot et al. 2012) (Exsiccatum: HIP:LIQ:2915).

Lecanoromycetes: Pertusariales

Coccotremataceae

Coccotrema coccophorum (Mont.) I. Schmitt, Messuti \& Lumbsch

Hábitat: corticícola. Registros en Magallanes: caleta Wilson, isla Clarence, archipiélago de Tierra del Fuego (Magallanes). Distribución en Chile: Valdivia a Magallanes (Quilhot et al. 2012). Distribución global: aparentemente endémica del sur de Sudamérica (Quilhot et al. 2012) (Exsiccatum: HIP:LIQ:3450).

\section{Coccotrema colobinum (Tuck.) Messuti}

Hábitat: zona de salpicaduras. Registros en Magallanes: bahía Ventisquero, estrecho de Magallanes (Magallanes). Distribución en Chile: Magallanes. Distribución global: Sudamérica (Fryday, 2007) (Exsiccatum: HIP:LIQ:14984).

Coccotrema cucurbitula (Mont.) Müll. Arg.

Hábitat: matorral enano turboso. Registros en Magallanes: isla Tammar, estrecho de Magallanes (Magallanes). Distribución en Chile: Malalcahuello a isla Navarino y Territorio Antártico (Quilhot et al. 2012). Distribución global: austral (Quilhot et al. 2012) (Exsiccatum: HIP:LIQ:4388).

Icmadophilaceae

Thamnolia vermicularis (Sw.) Schaer. subsp. vermicularis ( $\mathrm{Sw}$.) Schaer.

Sinonimia: Thamnolia vermicularis (Sw.) Schaer. Hábitat: sin información disponible. Registros en Magallanes: Chabunco (Magallanes). Distribución en Chile: Valdivia a isla Navarino (Quilhot et al. 2012). Distribución global: bipolar (Quilhot et al. 2012) (Exsiccatum: HIP:LIQ:15416*).

Lecanoromycetes: Teloschistales

Teloschistaceae

Josefpoeltia sorediosa S.Y. Kondr. \& Kärnefelt Hábitat: sin información disponible. Registros en Magallanes: laguna Timone 
(Magallanes). Distribución en Chile: el Panul (región Metropolitana) (Díaz \& López, 2017) a Magallanes. Distribución global: Sudamérica (Arup et al. 2013) (Exsiccatum: HIP:LIQ:3029*).

Polycauliona candelaria (L.) Frödén, Arup \& Søchting

Sinonimia: Xanthoria candelaria (L.) Th. Fr. Hábitat: sin información disponible.

Registros en Magallanes: laguna Timone (Magallanes). Distribución en Chile: Llanquihue a isla Navarino y Territorio Antártico (Quilhot et al. 2012). Distribución global: cosmopolita (Sancho et al. 1990); bipolar (Quilhot et al. 2012) (Exsiccatum: HIP:LIQ:3029*).

Lecanoromycetes: Umbilicariales

Umbilicariaceae

Umbilicaria nylanderiana (Zahlbr.) H. Magn.

Hábitat: sobre roca silícea expuesta en depresión húmeda. Registros en Magallanes: lago Grey, Parque Nacional Torres del Paine a 60 msnm (Última Esperanza). Distribución en Chile: desde Osorno a Magallanes y Territorio Antártico (Quilhot et al. 2012). Distribución global: bipolar (Quilhot et al. 2012) (Exsiccatum: HIP:LIQ:15365).

\section{DISCUSIÓN}

El presente trabajo constituye el primer catálogo de líquenes de la región de Magallanes. Está elaborado en torno a una colección biológica y la respectiva base de datos que resume información ecológica(e.g. macrositio, micrositio)delasdiferentes especies. Dicha información puede ser utilizada para consulta in situ por especialistas interesados. Pese a que el catálogo se complementa también con literatura actualizada, persisten algunos vacíos de información sobre la riqueza total de especies en la región, nicho ecológico, macroecología, relaciones biogeográficas y estado de conservación. Por ejemplo, Quilhot et al. (2012) registraron 319 especies en la región de Aysén, de las cuales 63 son compartidas con la región de Magallanes. No obstante, un catálogo definitivo aún es necesario. Aunque esta información contiene gaps tanto geográficos como ecológicos, se basa sobre un considerable conjunto de información geográfica en diversos sectores de Magallanes, donde también se incluyen numerosos ecosistemas (e.g. bosques caducifolios, bosques perennifolios, turberas, matorrales mesofíticos, matorrales xerofíticos, estepa patagónica, praderas naturalizadas, hábitat saxícolas, etc.). Según Galloway y Quilhot (1998), la flora liquénica en Chile consta de 1.383 taxa distribuidos en 304 géneros. Por lo que las especies registradas hasta ahora en Magallanes representan un bajo porcentaje de riqueza en comparación con la información existente a nivel país. Algunas de estas especies liquénicas se han clasificado según su estado de conservación, sin embargo, se reconoce la falta de antecedentes para categorizarlas correctamente y así revelar cifras más actuales y realistas a diferentes escalas geográficas (Quilhot et al. 1998).

\section{AGRADECIMIENTOS}

Agradecemos a los principales colectores (A. Elvebakk, E. Pisano, D. Lanfranco, O. Dollenz y E. Domínguez) y determinadores (A. Elvebakk, J. W. Bjerke, J. Redón y E. Pisano) que han contribuido a incrementar la colección del HIP y con ello, resguardar el patrimonio natural de la región de Magallanes. Nuestra gratitud para el Dr. Reinaldo Vargas, quien colaboró con la actualización de la información de la colección de líquenes del HIP.

\section{LITERATURA CITADA}

Aptroot, A., \& Sipman, H. (1991). New lichens and lichen records from New Guinea. Willdenowia, 20, 221-256.

Arup, U., Søchting, U., \& Frödén, P. (2013). A new taxonomy of the family Teloschistaceae. Nordic Journal of Botany, 31(1), 016-083.

Australian Biological resources study (2004). Flora of Australia, Vol. 56A, Lichens 4. Commonwealth of Australia, Canberra, Australia.

Calvelo, S., \& Fryday, A. M. (2006). New reports of lichens from Argentina Tierra del Fuego and the Falkland Islands (Islas Malvinas). The Bryologist, 109(3), 372-380.

Chesnokov, S., \& Konoreva, L. (2015). Additions to the lichen biota of SE Siberia: records from the Stanovoye Nagor'e Highlands (Trans- 
Baikal Region, Russia). Polish Botanical Journal, 60(2), 203-216.

Crespo, A., Divakar, P. K., Argüello, A., Gasca, C., \& Hawksworth, D. L. (2004). Molecular studies on Punctelia species of the Iberian Peninsula, with an emphasis on specimens newly colonizing Madrid. The Lichenologist, 36(5), 299-308.

Crous, P. W., Gams, W., Stalpers, J. A., Cannon, P. F., Kirk, P. M., David, J. C., \& Triebel, D. (2004a). An Online Database of Names and Descriptions as an Alternative to Registration. Mycological Research, 108(11), 1236-1238.

Crous, P. W., Gams, W., Stalpers, J. A., Robert, V., \& Stegehuis, G. (2004b). MycoBank: an online initiative to launch mycology into the 21st century. Studies in Mycology, 50(1), 19-22.

de Lange, P., Blanchon, D., Knight, A., Elix, J., Lucking, R., Frogley, K., Harris, A., Cooper, J., \& Rolfe, J. (2018). Conservation status of New Zealand indigenous lichens and lichenicolous fungi. New Zealand Threat Classification Series 27. Department of Conservation, Wellington.

Díaz, C., \& López L. (2017). Aportes al conocimiento de la biota liquenológica del matorral y bosque esclerófilo: estudio de la diversidad liquénica del parque público El Panul. Tesis de Pregrado. Universidad Metropolitana de Ciencias de la Educación, Santiago, Chile.

Divakar, P. K., Blanco, O., Hawksworth, D. L., \& Crespo, A. (2005). Molecular phylogenetic studies on the Parmotrema reticulatum (syn. Rimelia reticulata) complex, including the confirmation of $P$. pseudoreticulatum as a distinct species. The Lichenologist, 37(1), 55-65.

Elvebakk, A., \& Galloway, D. J. (2003). Notes on the heterogeneous genus Psoroma s. lat. New Zealand. Australasian Lichenology, 53, 4-9.

Elvebakk, A., Fritt-Rasmussen, J., \& Elix, J. A. (2007). The New Zealand lichen Pannaria leproloma (Nyl.) P. M. Jørg. and its panaustral relative $P$. farinosa nom. nov. The Lichenologist, 39(04), 349-359.
Elvebakk, A., Bjerke, J. W., \& Støvern, L. E. (2014). Parmelioid lichens (Parmeliaceae) in southernmost South America. Phytotaxa, 173(1), 1-30.

Estrabou, C., \& Adler, M. T. (1999). Novedades sobre Parmeliaceae sensu stricto (Ascomycota liquenizados) de la Provincia de Córdoba, República Argentina. Boletín de la Sociedad Argentina de Botánica, 34(12), 63-75.

Ferencová, Z., Cubas, P., Divakar, P. K., Molina, M. C., \& Crespo, A. (2014). Notoparmelia, a new genus of Parmeliaceae (Ascomycota) based on overlooked reproductive anatomical features, phylogeny and distribution pattern. The Lichenologist, 46(1), 51-67.

Fernández-Mendoza, F., \& Printzen, C. (2013). Pleistocene expansion of the bipolar lichen Cetraria aculeata into the Southern hemisphere. Molecular Ecology, 22(7), 1961-1983.

Fryday, A. M. (2007). Additional Lichen Records from New Zealand 47 Coccotrema corallinum Messuti and $C$. pocillarium (CE Cumm.) Brodo. Australasian Lichenology, 61, 3-5.

Galloway, D. J., \& Quilhot, W. (1998). Checklist of Chilean lichen-forming and lichenicolous fungi. Gayana Botánica, 55, 111-185.

Gatica, A., Pereira, I., \& Vallejos, O. (2011). Líquenes epífitos: una herramienta para estudiar la continuidad ecológica en Isla Mocha, Chile. Gayana Botánica, 68(2), 226-235.

Goffinet, B. (2012). Miniature forests of Cape Horn: ecotourism with a hand lens. University of North Texas Press.

Herrera, R. (2009). Primer inventario de levantamiento de información biológica en laguna Parinacota y Río ArabillaLíquenes, Tarapacá, Chile, Universidad Arturo Prat.

Kantvilas, G. (2016). Further observations on the lichen genus Megalaria in Tasmania: some species with blue-green apothecial pigments. Herzogia, 29(2), 421-435.

Kappen, L., Meyer, M., \& Bölter, M. (1988). Photosynthetic Production of the Lichen Ramalina terebrata Hook. f. et Tayl., in 
the Maritime Antarctic. Polarforschung, 58(2/3), 181-188.

Lindsay, D. C. (1974). The macrolichens of South Georgia. British Antarctic Survey Scientific Report, 89, 1-91.

Øvstedal, D. O., \& Gremmen, N. J. M. (2006). Lichens of sub-Antarctic Heard Island. South African Journal of Botany, 72(3), 353-366.

Passo, A., \& Calvelo, S. (2006). New reports and combinations in the family Pannariaceae (Lecanorales, lichenized Ascomycota). The Lichenologist, 38(6), 549-555.

Passo, A., \& Calvelo, S. (2011). Pannaria byssoidea (Pannariaceae), a new squamulose species from southern South America. The Bryologist, 114(4), 756-764.

Passo, A., Rodríguez, J. M., Chiapella, J. O., \& Messuti, M. I. (2018). The Antarctic lichen Cetraria subscutata is a synonym of Nephromopsis chlorophylla. The Lichenologist, 50(2), 239-245.

Pereira, I. (2007). Micobiota liquenizada del Parque Katalapi, X Región, Chile. Gayana Botánica, 64(2), 192-200.

Pereira, I., Müller, F., \& Moya, M. (2014). Influencia del pH de la corteza de Nothofagus sobre la riqueza de líquenes y briófitos, Chile central. Gayana Botánica, 71(1), 120-130.

Pisano Valdés, E. (1977). Fitogeografía de FuegoPatagonia chilena. I.-Comunidades vegetales entre las latitudes 52 y $56^{\circ} \mathrm{S}$. Anales del Instituto de la Patagonia, 8, 121-250.

Quilhot, W., Pereira, I., Guzmán, G., Rodríguez, R., \& Serey, I. (1998). Categorías de conservación de líquenes nativos de Chile. Boletín del Museo Nacional de Historia Natural, 47, 9-22.

Quilhot, W., Cuellar, M., Díaz, R., Riquelme, F., \& Rubio, C. (2010). Estudio preliminar de la flora liquénica de Isla Mocha, sur de Chile. Gayana Botánica, 67(2), 206-212.

Quilhot, W., Cuellar, M., Díaz, R., Riquelme, F., \& Rubio C. (2012). Lichens of Aisen, Southern Chile. Gayana Botánica, 69(1), 57-87.

Randlane, T., \& Saag, A. (2007). Cetrarioid lichens in the southern hemisphere - an identification key and distribution patterns of the species. Bibliotheca Lichenologica,
95, 489-499.

Redón, J. (1972). Líquenes de la región de Cachagua y Zapallar, Provincia de Aconcagua, Chile. Anales del Museo de Historia Natural de Valparaíso 5, 105-115.

Redón, J. (1985). Líquenes antárticos, Santiago, Chile, Instituto Antártico Chileno.

Redón, J., \& Quilhot, W. (1977). Los líquenes de Isla Navarino. I: Estudio sistemático y ecológico preliminar. Serie Científica Instituto Antártico Chileno, 5(1), 65-79.

Rodríguez, P., \& Printzen, C. (2014). Palicella, a new genus of lichenized fungi and its phylogenetic position within Lecanoraceae. The Lichenologist, 46(4), 535-552.

Rogers, R. W., \& Hafellner, J. (1988). Haematomma and Ophioparma: two superficially similar genera of lichenized fungi. The Lichenologist, 20(2), 167-174.

Rubio, C., Saavedra, M., Cuéllar, M., Díaz, R., \& Quilhot, W. (2013). Líquenes epífitos en el Parque Nacional Conguillío, sur de Chile. Gayana Botánica, 70(1), 66-81.

Sancho, L. G., Kappen, L., \& Schroeter, B. (1990). Primeros datos sobre la flora y vegetación liquénica de Isla Livingston (Islas Shetland del Sur, Antártida). En Actas del III Symposium Español de Estudios Antárticos. Comisión Interministerial de Ciencia y Tecnología, Madrid.

Saucedo, C. (2004). Plan de Manejo Reserva Nacional Lago Cochrane (Tamango) y lotes aledaños (Documento trabajo $\mathrm{N}^{\circ}$ 406), Coyhaique, Chile, Corporación Nacional Forestal (CONAF).

Schumm, F., \& Elix, J. A. (2014). Images from Lichenes Australasici Exsiccati and of other characteristic Australasian Lichens. (Vol. 1). Norderstedt.

Sehnal, L. (2012). Photosynthesis of lichens from Antarctic Peninsula and South Patagonia in relation to environmental factors (bachelor thesis). Masaryk University, Department of Experimental Biology, Brno, Czech Republic.

Skottsberg, C. J. F. (1941). Lista sistemática de musgos y líquenes chilenos. Boletín del Museo Nacional de Historia Natural, 19, 113-132. 
Spielmann, A. A., \& Marcelli, M. P. (2008). Punctelia (Parmeliaceae, lichenized Ascomycota) from roadsides and slopes in the Serra Geral of Rio Grande do Sul, Brazil. Biociências, 16(2), 79-91.

Syrek, M., \& Kukwa, M. (2008). Taxonomy of the lichen Cladonia rei and its status in Poland. Biología, 63(4), 493-497.

Vergara, C., \& Reyes, R. (2000). Diagnóstico y propuesta de incorporación del sitio prioritario "Cordillera Pelada" al Sistema Nacional de Áreas Silvestres Protegidas del Estado (Informe Técnico $N^{\circ} 1$ ), Valdivia, Chile, Programa Ecorregión Valdiviana, WWF.
Villagra, J., Montenegro, D., San Martín, C., Ramírez, C., \& Álvarez, I. (2009). Estudio de la flora liquénica de las turberas de la Comuna de Tortel (región de Aisén), Patagonia chilena. Anales del Instituto de la Patagonia 37(1), 53-62.

White, F. J., \& James, P. W. (1988). Studies on the Genus Nephroma II. The Southern Temperate Species. The Lichenologist, 20(2), 103-166.

Youn, U. J., So, J. E., Kim, J. H., Han, S. J., Park, H., Kim, I. C., \& Yim, J. H. (2018). Chemical constituents from the Antarctic lichen, Stereocaulon caespitosum. Biochemical Systematics and Ecology, 80, 73-75. 